\title{
Empreendedorismo digital: um estudo sobre o uso da tecnologia como geração de negócios nas startups educacionais*
}

\author{
Digital entrepreneurship: a study on the \\ use of technology as a business generation in \\ educational startups
}

\section{Alexandre Albuquerque Domingues ${ }^{1}$ Kathryn Floyd-Wheeler ${ }^{2}$ Neide Silva Nascimento ${ }^{3}$}

* Recebido em: $16 / 11 / 2016$. Aprovado em: 23/03/2017.

1 Graduação em Gestão de Tecnologia e Sistemas de Informação pela Universidade Nove de Julho (2008), Especialista em Formação de Docentes para o Ensino Superior pela Universidade Nove de Julho(2010); revisor dos seguintes periódicos: Revista Universitas Gestão e TI, Revista de Gestão da Tecnologia e Sistemas de Informação, Revista de Administração da Universidade Federal de Santa Maria (UFSM) e Gestão Contemporânea. Atualmente é diretor administrativo e fundador (owner) - Espaço Educacional LTDA. Tem experiência na área de Ciência da Informação, com ênfase em Gestão de Tecnologia da Informação, atuando principalmente nos seguintes temas: sistema de gestão administrativa, banco de dados e sistemas de informação.

2 Bachelor's at Economics from University of New South Wales (1996), master's at Economics from University of New South Wales (2004) and doctorate and Ph.D at Economics from University of New South Wales (2008). Has experience in Probability and Statistics, focusing on Probability and Statistics

3 Graduada em Ciências da Computação pela Universidade Metodista de São Paulo (2006). Atualmente leciono como professora no Ensino Superior leciono na Faculdade Anhanguera (Unidade de São Bernardo do Campo) nas disciplinas ligadas a desenvolvimento de softwares e websites. No curso técnico leciono no Centro Estadual de Educação Tecnológica Paula Souza e no Colégio Anchieta. Como freelance realizo a criação de sites comerciais.

\section{Resumo}

Este artigo versa sobre como a tecnologia da informação (TI) influência no aumento da qualidade da atividade empreendedora. Devido à nova realidade brasileira que se apresenta em um universo complexo e multifacetado, acaba exigindo grande flexibilidade do empreendedor para se conectar com o cliente por meio de aplicativos e de dispositivos móveis que passam a ser acessíveis à sociedade brasileira, garantindo flexibilidade e novas relações de negócios em atividades tradicionais como a educação ou outras atividades do terceiro setor . Nesse sentido o estudo objetiva explicar como a política de tecnologia da informação auxilia na melhoria dos serviços em uma organização educacional. O método "Startup" foi utilizado como objeto de estudo e validou o aumento da produção dos serviços educacionais por meio da publicação de aplicativos propostos na ESPEDUC, dessa forma, a meta de divulgação para oferta e demanda de publico foi atingida por meio de uma política de geração de produtos e acessos móveis digitais a futuros e atuais clientes.

Palavras-chave: Empreendedorismo. Tecnologia da Informação. Startup.

\section{Abstract}

This article is about how information technology (IT) influences on increasing the quality ofentrepreneurial activity. Because the new Brazilian reality presented in a complete and multifaceted universe, just requiring great flexibility entrepreneur to connect with customers through mobile applications and devices that become accessible to Brazilian society, ensuring flexibility and new business relationships in traditional activities such as education or other activities of the third sector. In this sense the study aims to explain how information technology policy helps to improve services in an educational organization. Based on quantitative research method "Startup" was used as an object of study and validated the increased production of educational services through the publication of proposed applications in ESPEDUC, so the goal of disclosure for public supply and demand was achieved through a product generation policy and digital mobile access to current and future customers.

Keywords: Entrepreneurship. Information Technology. Startup. 


\section{Introdução}

O Empreendedorismo no Brasil ganha notoriedade com o surgimento do terceiro setor desde nos anos 1990. As atividades empreendedoras, com o advento e ampliação da tecnologia da informação, nas décadas seguintes, permitem que fatores como distância entre a empresa e o cliente diminuem, gradativamente, e, em contrapartida, também, permitem uma maciça exposição de marcas e produtos_em qualquer lugar e em qualquer momento por meio de dispositivos móveis, em especial o telefone celular, que hoje é uma vitrine de serviços muito poderosa. Nesse paradigma radicalmente construído, as organizações têm que lidar com essa realidade de relacionamento a ser desenvolvida, atividades tradicionais como conhecemos passam por essa modificação social e criam um novo "modus-operanti" para a geração de negócios. Para que haja essa sincronia de eventos, é necessária a implantação de um arcabouço de ferramentas e técnicas que seja capaz de coordenar e auxiliar os serviços de TI para que estes tenham uma qualidade elevada (SALLÉ, 2004).

Nesse sentido o presente artigo tem como questão de pesquisa: mensurar o aumento nas vendas de serviços educacionais por meio dos dispositivos de tecnologia da informação? Por meio de pesquisa quantitativa, com uma base de dados aplicada na "ESPEDUC", estima-se analisar o percentual efetivo de clientes firmados. O trabalho contribuirá na prática para outras empresas possam adaptar seus modelos de negócios a ferramentas tecnológicas e possam aperfeiçoar seus lucros e serviços consideravelmente.

Neste artigo, também, será analisada a forma que a divulgação dos serviços de educação oferecidas no "startup" em questão por ferramentas como "Google Adwords" e serviços de "e-mail" contribui, de maneira definitiva, para a prospecção de novos clientes.

A próxima seção fornecerá uma sinopse da literatura tradicional sobre gerenciamento de mudanças, qualidade de serviços e empreendedorismo. A terceira seção descreve o método utilizado e os procedimentos. A seção subsequente descreve a intervenção realizada, bem como os mecanismos adotados. A quinta seção aponta os resultados obtidos com a intervenção. A seção final aborda as implicações gerenciais e acadê-

\section{Referencial Teórico}

A qualidade em serviços vem sendo estudada com maior profundidade nos últimos 30 anos, considerada como aspecto importante e, até mesmo um subcampo do Marketing de Serviços (GRÖNROOS, 1982). A busca pelo entendimento da avaliação da qualidade existe faz muito tempo, trata-se de um conceito de difícil avaliação quando comparada à qualidade de bens, conforme constatam. Grönroos (1982) traduz a qualidade percebida como uma comparação entre a qualidade experimentada pelo consumidor e a qualidade experimentada na visão da empresa prestadora de serviços, além de ressaltar a existência de duas tipologias: (1) Qualidade Técnica, que envolve o que o consumidor de fato recebe com o serviço e (2) Qualidade Funcional, que abrange a forma pela qual o serviço é entregue.

Em outra perspectiva, Lehtinen e Lehtinen (1982) postulam três dimensões para a qualidade em serviços: (1) Qualidade Física, inclui os aspectos físicos do serviço; (2) Qualidade Corporativa, relativa a aspectos como a imagem e o perfil da empresa e (3) Qualidade Interativa, que deriva das interações entre a empresa e os consumidores, assim como dos consumidores entre si. Lewis e Booms (1983) definem a qualidade em serviços como sendo a conformidade com as expectativas dos consumidores e Babakus e Boller (1992) acreditam que é necessário utilizar, apenas, uma forma de avaliar os serviços por meio da percepção do resultado de um processo cognitivo dos consumidores.

A qualidade é definida por Cronin Jr e Taylor (1992) como antecedente da satisfação e acreditam que a qualidade poderia ser diferente em diferentes mercados e indústrias, não sendo possível generalizá-la para todos os setores da indústria. A importância da construção da qualidade do serviço reside na sua conceitualização como a avaliação do consumidor de um serviço e as relações supostas, portanto, com outras construções, como satisfação, lealdade e, finalmente, competitividade e lucratividade (SMITH; REYNOLDS, 2002) enquanto Cáceres e Paparoidamis (2007) argumentam que a percepção da qualidade do serviço é um resultado de uma comparação entre o que cliente considera como o serviço deveria ser e suas percepções sobre o desempenho real oferecido pelo prestador de serviços. 


\section{Metodologia}

Para a concretização deste relato técnico, foi utilizada a técnica estatística Análise Discriminante, que, segundo Maroco (2003, p. 331), é uma técnica estatística multivariada de dados cujos objetivos são:

a) identificação das variáveis que melhor discriminam entre dois ou mais grupos de indivíduos estruturalmente diferentes e mutuamente exclusivos;

b) a utilização destas variáveis para criar uma função discriminante que represente de forma parcimoniosa as diferenças entre os grupos;

A "ESPEDUC" foi concebida no formato "Startup" para oferecer produtos e serviços educacionais, entre os quais Ensino Fundamental e Médio, além de treinamentos corporativos a empresas e indústrias da grande Porto Alegre-RS; os dados foram coletados em uma organização cliente na qual manteremos seu anonimato e trataremos como "empresa-cliente" da ESPDUC, esta é uma empresa de médio porte que oferece como vantagens e benefícios aos seus colaboradores serviços educacionais aos que têm filhos e responsáveis. A coleta de dados foi realizada mediante entrevista semiestruturada contemplando o universo de 80 (oitenta funcionários) em 2016 com variação etária entre dezoito (18) e trinta e cinco anos (35) respectivamente.

De acordo com Hair et all (2005, p. 209), “[...] a discriminação é conseguida estabelecendo-se os pesos da variável estatística para cada variável, para maximizar a variância entre grupos relativa à variância dentro dos grupos, e a função discriminante é determinada de uma equação."

A equação assume a seguinte forma:

Gráfico 1: Equação estatística da pesquisa.

\begin{tabular}{|l|}
\hline \multicolumn{1}{|c|}{$\mathbf{Z}_{\mathrm{jk}}=a+\mathbf{W}_{1} \mathbf{X}_{1 \mathrm{k}}+\mathbf{W}_{2} \mathbf{X}_{2 \mathrm{k}}+\ldots+\mathbf{W}_{\mathrm{n}} \mathbf{X}_{\mathrm{nk}}$} \\
\hline Onde: \\
$\mathbf{Z}_{\mathrm{ik}}=$ escore $Z$ discriminante da função discriminante j para o \\
objeto $\mathrm{k}$. \\
$\mathbf{a}=$ intercepto. \\
$\mathbf{W}_{1}=$ peso discriminante para a variável independente 1. \\
$\mathbf{X}_{1 \mathrm{k}}=$ variável independente 1 para o objeto $\mathrm{k}$. \\
\hline
\end{tabular}

Fonte: Elaborado pelos autores.

A entrevista semiestruturada foi aplicada sob a determinação de três variáveis detectadas no cenário:
Tabela 1 - Variáveis da Pesquisa

\begin{tabular}{llll}
\hline Variáveis & Indicadores & Código \\
\hline $\begin{array}{llll}\text { Indepen- } \\
\text { dentes }\end{array}$ & $X^{1}$ & Funcionário tem filhos e benec. Educ. & \\
& $X^{2}$ & Funcionário n/filhos e sem benec educ & \\
Dependentes & $X^{3}$ & $\begin{array}{l}\text { Funcionário teve acesso ao portfólio de } \\
\text { serviços/Startup }\end{array}$ & 1 -Celular \\
& & & $2-$ Computador \\
\hline Fonte: Elaborado pelos autores.
\end{tabular}

Fonte: Elaborado pelos autores.

A classificação dos indicadores, anteriormente descrita, se fez necessária, pois, de acordo com Hair (2005, p. 221), as observações atípicas podem ter um impacto na classificação de quaisquer resultados da análise discriminante.

Para estabelecer a variável dependente $\left(\mathrm{X}^{3}\right)$, considerou-se a seguinte situação: o funcionário da empresa-cliente tem filhos e acesso ao pacote educacional e recebe informativos da "ESPEDUC" através de celular ou computador. Portanto, para os resultados do indicador abaixo foram atribuídos códigos um (1) para acesso celular e dois (2) para computador.

A análise discriminante foi efetuada no software "IBM-SPSS ${ }^{\oplus}$. - Statistical Packages for Social Sciencies versão 13.0, utilizado como ferramenta para execução de testes estatísticos a um nível de significância de 0,05 .

\section{Resultados Obtidos e Analise}

Após a execução da análise discriminante, o primeiro passo consistiu na análise preliminar das variáveis independentes, verificando a existência de diferenças entre as médias. Maroco (2003, p. 351) testou a hipótese de que as médias dos grupos são iguais, em que, entre as variáveis, pelo menos, em um grupo as médias são diferentes; nesse caso, o objetivo é rejeitar a hipótese nula. Dessa forma, as hipóteses testadas foram as seguintes:

$\mathrm{H}^{\mathrm{o}}=$ média dos dois grupos são iguais

$\mathrm{H}^{1}=$ média dos grupos são diferentes.

O resultado desse teste está na Tabela 1, nota-se que, apenas, as variáveis $\mathrm{X}^{1}$ e $\mathrm{X}^{2}$ não rejeitam a hipótese nula $\left(\mathrm{H}^{\circ}\right)$, pois o $p$-value (sig.) é menor que o (nível de significância) de 0,05. Com isso, não aceita $\mathrm{H}^{\circ}$, concluindo que a função discriminante é altamente significativa. 
Tabela 2 - Lambda de Wilks

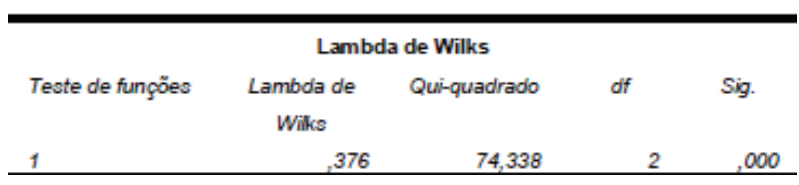

Fonte: Resultado da análise discriminante pelo software SPSS ${ }^{\oplus}$.

A tabela 3 evidencia o valor do ou autovalor, que, segundo Maroco (2003, p. 353), é uma medida relativa de quão diferente os grupos são na função discriminante. Nesse caso, o resultado é de, apenas, uma função, correspondendo a 100\% da variância explicada em termos de diferenças entre grupos. Essa tabela, também, apresenta a correlação canônica, que demonstra o nível de associação entre os escores discriminantes e os grupos, para utilizar este resultado como \% da variável dependente explicada pelo modelo, de acordo com Hair 2005, p. 238), deve-se elevar o resultado da correlação ao quadrado, no caso, tem se $R^{2}=(\mathbf{0 , 7 9 0})=\mathbf{1 , 5 8 0}$, ou seja, a função explica $90 \%$ da discriminação entre os grupos.

Tabela 3 - Valores Próprios

\begin{tabular}{|c|c|c|c|c|}
\hline \multicolumn{5}{|c|}{ Valores próprios } \\
\hline Função & Autovalor & $\begin{array}{c}\text { \% de } \\
\text { variância }\end{array}$ & $\%$ cumulativa & $\begin{array}{l}\text { Correlação } \\
\text { canónica }\end{array}$ \\
\hline 1 & $1,659^{2}$ & 100,0 & 100,0 & ,790 \\
\hline
\end{tabular}

A Função um, funções discriminantes canônicas foram usadas na análise

Fonte: Resultado da análise discriminante pelo software SPSS ${ }^{\circledR}$.

A próxima tabela (tabela 4) apresenta as variáveis selecionadas para compor a função e seus respectivos coeficientes padronizados.

Tabela 4 - Coeficientes de funções discriminantes canônicas padronizados

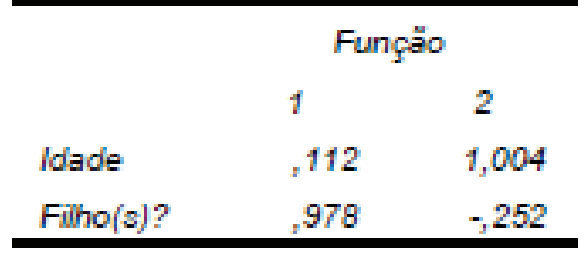

Standardized coefficients

Fonte: Resultado da análise discriminante pelo software SPSS ${ }^{\oplus}$.

Os tamanhos de grupos usados no cálculo precedente são baseados no conjunto de dados empregado na amostra de análise e não incluem a amostra de validação. O procedimento para classificar os tipos de a) - Classifica-se um funcionário sem filhos se seu escore discriminante for menor que 0,664 ;

b) - Classifica-se um funcionário com filhos se seu escore discriminante for maior que 0,664 .

Tabela 5 - Funções em Centoides de Grupo

\begin{tabular}{lcc}
\hline Portifólio da Empresa & \multicolumn{2}{c}{ Funçäo } \\
& 1 & 2 \\
1 &, 167 &,- 264 \\
2 &, 913 &, 179 \\
3 & $-2,641$ &, 119 \\
\hline
\end{tabular}

Funções discriminantes canônicas não padronizadas avaliadas em médias de grupo

Fonte: Resultado da análise discriminante pelo software SPSS ${ }^{\circledR}$.

\section{Conclusões e Considerações Finais}

O empreendedorismo como outras atividades de mercado sofrem transformações inclusive o meio de comunicação se torna algo indispensável para o empreendedor poder avaliar seus mecanismos de abordagem ao mercado que se propõe a atuar.

Domingues (2015) enfatiza que: [...] O Valor Empresarial dos sistemas integrados se resume em aumentar a eficiência operacional, e oferecer à empresa amplas informações para apoio à tomada de decisão e viabilizam respostas rápidas às solicitações de informação ou produtos feitas por clientes [...].

Nesse contexto, considerando os resultados gerais da análise discriminante, depois que os pressupostos foram atendidos, observa-se que as variáveis selecionadas são significativamente, portanto, devemos analisar novos mecanismos de controle e relacionamento com clientes e os dispositivos móveis em especial celulares principalmente mostram-se como um novo "status" para o mercado de uma sociedade "instantânea" e "conectada", como empreendedores devemos levar em consideração que as pessoas cada vez mais buscam terceirizar seu tempo e necessidade por meio de mecanismos como o "aplicativo móvel"; em que deixa de ser uma tendência e passa a se tornar realidade impactando no relacionamento com o cliente.

\section{Referências}

BABAKUS, E.; BOLLER, G. W. An empirical assessment of the SERVQUA scale. Journal of Business Research, v. 24, n. 3, p. 253-268, 1992. 
BIANCOLINO, C. A. et al. Protocolo para elaboração de relatos de produção técnica. Revista Gestão e Projetos, v. 3, n. 2, p. 294-307, 2012.

CACERES, R. C.; PAPAROIDAMIS, N. G. Service quality, relationship satisfaction, trust, commitment and business-to-business loyalty. European Journal of Marketing, v. 41, p. $836-867,2007$.

CRONIN JR, J. J. TAYLOR, S. A. Measuring service quality: a reexamination and extension. The Journal of Marketing, p. 55-68, 1992.

DOMINGUES, A. A. et al. Gestão estratégica de tecnologia da informação: estudo sobre a aplicação da TI como suporte de decisão as organizações. In: CONTECSI - FEA/USP - Conferência Internacional sobre Sistemas de Informação e Gestão de Tecnologia, 12., 2015, São Paulo. Anais... São Paulo, 2015. p. 1432-1448.

DOMINGUES, A. A.; WALKER, E.; FLOYD-WHEELER, K. Governança de tecnologia da informação: estudo sobre a relação entre a T.I e a governança corporativa. In: CONTECSI - FEA/USP - Conferência Internacional sobre Sistemas de Informação e Gestão de Tecnologia, 13., 2016, São Paulo. Anais... São Paulo, 2016. p. 195-206.
GRÖNROOS, C. Service quality: the six criteria of good perceived service. Review of Business, v. 9, n. 3, p. 10, 1988.

GRÖNROOS, C. Strategic management and marketing in service sector. Cambridge, MA: Marketing Science Institute, 1982.

HAIR, Joseph F. et al. Análise multivariada de dados. Porto Alegre: Bookman, 2005.

LEHTINEN, U.; LEHTINEN, J. R. Service quality: a study of quality dimensions. Service Management Institute, 1982.

LEWIS, R. C.; BOOMS, B. H. The marketing aspects of service quality. Emerging Perspectives on Services Marketing, v. 65, n. 4, p. 99-107, 1983.

MAROCO, J. Analise estatística com a utilização do SPSS. Lisboa: Silabo, 2003.

SALLÉ, M. IT service management and IT governance: review, comparative analysis and their impact on utility computing. Hewlett-Packard Company. p. 8-17, 2004. 